\title{
FORTALECIMIENTO DEL DESEMPEÑO DE LOS NIÑOS DE PRIMERO DE PRIMARIA EN LA RESOLUCIÓN DE PROBLEMAS DE ESTRUCTURA ADITIVA: CAMBIO Y COMBINACIÓN
}

\author{
Jessica Andrea Rangel Muñoz \\ Licenciada en pedagogía infantil \\ Fundación Universitaria los libertadores. \\ jeanramu1707@hotmail.com
}

\author{
María del Pilar García Chitiva \\ Magíster en Educación \\ Doctoranda en Educación \\ Fundación Universitaria los libertadores. \\ jeanramu1707@hotmail.com
}

\section{Cómo citar este artículo:}

Rangel, J.A. y García, M.P. (2014). Fortalecimiento del desempeño de los niños de $1^{\circ}$ primaria en la resolución de problemas de estructura aditiva: cambio y combinación. Espiral, Revista de Docencia e Investigación, 4(2), 63 - 82

\begin{abstract}
Resumen
A través del presente artículo de investigación se intenta demostrar que el aprendizaje de las matemáticas, requiere de un proceso sistematizado de enseñanza teniendo en cuenta el desarrollo cognitivo del individuo. De la misma manera, cabe señalar, que aunque el individuo cuente con predisposiciones innatas, necesita de la interacción social para poderlas potencializar. Por lo tanto, según Vygotsky, cuando se enseña matemáticas, lo ideal es que el maestro sea un mediador ante el conocimiento que aún no logra alcanzar el sujeto, recurriendo a estrategias de andamiaje, en la cuales se evidencie el apoyo entre ambos. Para este estudio, se utilizó un diseño cuasi experimental, que contó con la participación de 26 estudiantes para el grupo control y 25 del grupo experimental, en el grado de primero de primaria, quienes realizaron un pre y postest, para analizar el estado inicial en el desempeño de resolución de problemas y su percepción hacia las matemáticas; y por otra parte la incidencia que tuvo la intervención pedagógica. Se pudo determinar que la utilización del lenguaje matemático en la comunicación de los sistemas de notación simbólica, la capacidad para la argumentación y la realización de conjeturas en el desarrollo de un problema, fortaleció de manera transversal otras habilidades como la lectura y la escritura.
\end{abstract}

Palabras clave: Matemáticas, apoyo, resolución de problemas, construcción de número, Sistema Numérico Decimal.

\footnotetext{
Abstract

This research paper seeks to show that learning mathematics requires a systematic process of education considering the cognitive development of the person. Likewise, it should be noted that although the person
}

has innate predispositions, he needs social interaction so that these predispositions can be strengthened. Therefore, according to Vygotsky, when teaching mathematics, it would be grateful that the teacher would be a mediator between the person and the knowledge that hasn't been reached yet by using scaffolding strategies, in which there is evidence of support among both. For this research, a quasi-experimental design was used, which included the participation of 26 students for the control group and 25 for the experimental group in the first elementary level; these studentes carried out a pre and post-test to analyze their initial state for problem solving and their perception towards mathematics. Besides, the impact that the pedagogical intervention had on it. It was possible to determine that the use of mathematical language in communicating symbolic notation systems, the capacity for reasoning and making guesses when solving a problem, strengthened transversely other skills such as reading and writing.

Keywords: Mathematics, support, problem solving, building number, Decimal Number System.

\section{Introducción}

De acuerdo con los resultados de las pruebas Pisa realizadas en el año 2009, en las que se evalúa el rendimiento académico de 65 países, entre los resultados obtenidos, Colombia se encuentra en el puesto 52 en lectura, 58 en matemáticas y 54 en ciencias. En términos generales el $71 \%$ de los estudiantes no cuenta con el desempeño mínimo establecido (García, 2011).

De igual manera, en el documento de la alcaldía mayor de Bogotá (2011), Lo que dicen los 
resultados de las pruebas externas en Bogotá de la Secretaria de Educación del Distrito se pone en evidencia en la Prueba Comprender adelantada en el año 2010 que los estudiantes del sector oficial tienen un promedio de 48,55 y el sector privado 55,86 . Asimismo en esta prueba consideró que los estudiantes del sector oficial se encuentran en un nivel bajo en relación a los del sector privado en la compresión de la información que implica realizar operaciones de matemáticas básicas (suma y resta). Del mismo modo en las Pruebas Saber del grado $5^{\circ}$ de la ciudad de Bogotá del año 2013, en cuanto al desempeño en el área de matemáticas, en el documento de Bogotá cómo vamos. Indicadores de calidad del 2012, muestran que el 19\% de los estudiantes tiene un desempeño insuficiente; el $32 \%$ tiene un desempeño mínimo; el $28 \%$ tiene un desempeño satisfactorio y el $20 \%$ tiene un desempeño avanzado. En este sentido, algunos autores hacen referencia a algunos factores que inciden en la raíz del problema.

Kammi (2000) citando a Piaget, determina que existen tres tipos de conocimiento: de tipo físico, social y lógico matemático. El conocimiento físico que hace referencia a los objetos de la realidad exterior, a las cualidades y características que los describen. El conocimiento social hace referencia a todos los conocimientos que son por transmisión social como el lenguaje. Por otro lado, el conocimiento lógico matemático se define desde las relaciones construidas por cada individuo. Entiéndase relación como la "creada mentalmente por el individuo" (Kammi, 2000). Por tanto, Kammi (2000, p.56) argumenta que "la aritmética se enseña por transmisión como si fuera un conocimiento social" esto quiere decir, que en la enseñanza de la aritmética no se tiene en cuenta las relaciones creadas por el niño sino que se tiene presente si su respuesta es correcta o no y esto, como dice la autora, contribuye a que el niño pierda su iniciativa y su confianza en su propia capacidad, por consiguiente, algunos estudiantes perciben las matemáticas como incompre-

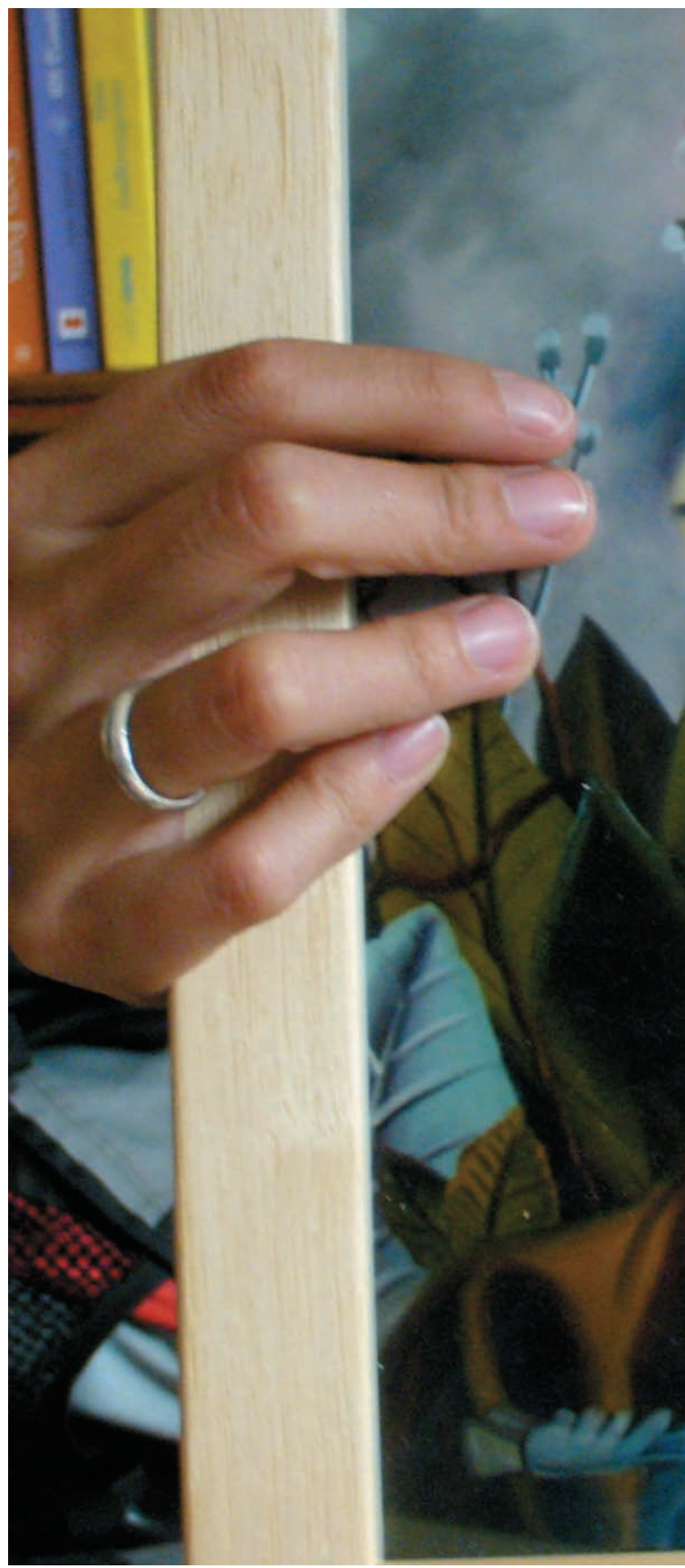


sibles. Por otro lado, algunos educadores, centran el proceso de enseñanza, en "los hechos de adición" o en la enseñanza de técnicas para adquirir hechos numéricos, y no en la acción mental de los niños y su nivel de maduración.

Por tal motivo, afirma Baroody (2005) que la "enseñanza no se adopta a la manera de pensar de los niños" por lo cual, en algunas situaciones en los procesos de enseñanza y aprendizaje, se pretende que los estudiantes, solo memoricen de manera rígida y sin sentido los algoritmos, de tal manera, que no se tenga presente las necesidades e intereses de los estudiantes, y de la misma forma, el sentido, tanto social como cultural de las matemáticas. Es por ello que el autor afirma que los niños pierden "deseo y la motivación de estudiar" porque creen que no pueden responder al ritmo que se exige, y por tal razón, se sienten incapaces.

Como anteriormente se mencionó, los estudiantes pierden el interés la motivación, y en consecuencia hace que en ocasiones consideren difícil las matemáticas, por lo cual es indispensable que se analicen posibles mecanismos de solución que permitan evidenciar un progreso en la realidad actual del país, en el desempeño académico en especial en matemáticas desde el grado primero.

El aprendizaje de las matemáticas, requiere de un proceso sistematizado de enseñanza que permita articular los diferentes conceptos y relaciones numéricas en la compresión del individuo. Cada sujeto cuenta con predisposiciones innatas, que hacen posible tener una estructura base, para conformar representaciones, sin embargo, es necesario el ambiente para poderlas potencializar.

Moll (1993) citando a Vigotsky, afirmó que en su teoría sociocultural, da cuenta de la importancia de características sociales y culturales del individuo para potenciar su desarrollo. Por lo tanto, se determina que existe una zona de desarrollo próximo, que la define como
La distancia entre el nivel real de desarrollo, determinado por la capacidad de resolver independiente un problema y el nivel de desarrollo potencial, determinado a través de la resolución de problema bajo la guía de un adulto o en colaboración con otro compañero más capaz. (p.86)

Podría decirse que mientras un estudiante esté en un estado inicial de desarrollo y que mientras tenga, sea un maestro u otro par más capacitado, puede avanzar en su desarrollo cognitivo. Maza (1995), en relación a la teoría de Vigostky, afirma que en la aritmética resulta importante cómo el sujeto construye el conocimiento a partir del compartir con el docente o tutor, a esta interacción la denominó conocimiento inter psicológico. Mediante el proceso de asimilación el sujeto lo trasforma en conocimiento interno o conocimiento intra- psicológico. Es decir, mientras haya un constate apoyo entre el maestro y el estudiante, o un estudiante y otro más capaz, puede haber una mayor asimilación del conocimiento. En otras palabras, lo que antes no entendía el estudiante, por medio de una ayuda y modelación, lo comprendió y luego, por si solo lo pudo poner en práctica.

Maza (1995) citando a Wersch (1985), hace referencia a un proceso que permite comprender con mayor precisión el transcurso del conocimiento inter psicológico al conocimiento intra psicológico, siendo así que, se resaltan los siguientes pasos: a) no hay una comunicación adecuada entre maestro y estudiante, b) el niño y maestro empiezan a compartir, pero no comprende aún las acciones del maestro en relación a un problema, c) en este nivel hay un concordancia de representaciones entre ambos, es decir, ambos comprenden las acciones que se deben hacer, d) el niño por si solo puede resolver el problema, sin la necesidad de pedir ayuda o ponerse de acuerdo con el maestro.

Cascallana (1999) en congruencia con la teoría de Vygotsky determina que "el niño que contrasta sus soluciones con las de otros com- 
pañeros, se ve obligado a modificar sus esquemas, a encontrar otras soluciones más acordes a la realidad, potenciándose así el desarrollo cognitivo" (p.16), y por lo cual, hace posible que el estudiante cuando se relaciona con el profesor o con otros pares, tenga otros referentes que hacen posible un proceso de asimilación/ acomodación. Mientras más contacto tenga el niño con otras personas va a cambiar y modificar sus esquemas previos y a reestructurarlos a unos nuevos que permitan resolver el problema con mayor facilidad.

Con el propósito de fortalecer el desempeño de la resolución de problemas matemáticos de estructura aditiva específicamente los de cambio y combinación, es necesario que el sujeto tenga unas bases o insumos necesarios, como la consolidación en la construcción de número, la compresión del sistema numérico decimal y bagaje de experiencias en resolución de problemas desde lo real, figurativo y no figurativo.

Por otro lado, Echenique (2006), determina las variables que se manifiestan desde la definición del problema en relación a la diferencia que este tiene con un ejercicio. Se toma como referencia el cuadro de la autora donde presenta dicho contraste entre problema y ejercicio.
Con respecto a la tabla 1 es necesario dar cuenta que el ejercicio no es un problema, ya que lo realiza mecánicamente, es decir, el estudiante no acude a emplear diferentes estrategias para solucionarlo sino que solamente ya sabe que tiene que hacer. De tal manera, que no es emocionante sino que se torna aburrido. Además el ejercicio no es significativo para el estudiante, pues no implica que sea necesario emplear saberes y conocimientos previos sino solo la operación que corresponde. Por lo cual la presentación de un problema que sea significativo, que motive al estudiante, que permita colocar en discusión la estrategias empleadas y que haga posible que los resultados sean propios, que no sea tan fácil para el estudiante sino que sea un reto pero que tampoco sea tan difícil, sino que se adapte a la formación y al desarrollo del estudiante.

A partir de lo anterior, se diseñó una propuesta de intervención, que permitiera que los estudiantes de primero de primaria, en primera instancia, afianzaran sus habilidades en la resolución de problemas en particular los problemas de estructura aditiva de cambio y combinación; y en segunda instancia, fomentar una percepción positiva hacia las matemáticas.

Tabla 1. Diferencia entre ejercicio y resolución de problemas

\begin{tabular}{|c|c|}
\hline EJERCICIO & PROBLEMAS \\
\hline Se realiza de forma mecánica & Supone un reto \\
\hline Se resuelve en un tiempo corto & Requiere más tiempo para la resolución \\
\hline No es contextualizado & Tiene relación al contexto socio cultural de la persona \\
\hline Generalmente tiene una sola solución & Puede presentarse una o varias solución y las vías para llegar a ellas puede ser variadas \\
\hline Son numerosos en los libros & No hay mucho \\
\hline $\begin{array}{l}\text { No hay lazos especiales entre la persona } \\
\text { que lo resuelve y el ejercicio }\end{array}$ & $\begin{array}{l}\text { Se hace emocionante ya que la situación es desconocida por la persona y la cual } \\
\text { motivara para la búsqueda perseverante de la solución y además de satisfacción de } \\
\text { encontrar la respuesta }\end{array}$ \\
\hline $\begin{array}{l}\text { La finalidad es la aplicación mecánica de } \\
\text { algoritmos }\end{array}$ & $\begin{array}{l}\text { La finalidad es ahondar en los conocimientos y experiencias que se poseen que se } \\
\text { hagan útiles en la solución }\end{array}$ \\
\hline
\end{tabular}

Fuente: Echenique, U. I. (2006), Matemáticas Resolución de Problemas, Educación Primaria. Gobierno de Navarra. Departamento de Educación. Recuperado de www.cfnavarra.es/publicaciones. 
Según Maza (1989) y Echenique (2006) los problemas se clasifican según las acciones y las relaciones que enuncian los ejercicios, por lo cual que se presentan los siguientes:

1. Cambio: hace referencia a un problema que tiene una cantidad inicial que se modifica en el tiempo y da lugar a otra. Cl-M-CF.

2. Combinación: relación de dos conjuntos que unidos forman un todo. P1 P2-T.

3. Comparación: comparativo de superioridad o de inferioridad entre dos cantidades.

4. Igualación: una de las cantidades debe modificarse para igualar a otra cantidad.

Sin embargo, Maza (1989), indica que los problemas de estructura aditiva tiene un nivel de dificultad, siendo propicio que se le trabaje con tipo cambio, luego combinación y posteriormente comparación. En este proyecto de investigación, solo se tratan problemas de tipo cambio y combinación porque son los más apropiados para esta edad.

Para el logro de los propósitos que se plantearon, se dispuso de un Sistema de Apoyo Pedagógico que, de manera estructurada y organizada, tuvo lugar en 3 etapas: la primera analizar el desempeño inicial de los estudiantes en la resolución de problemas de estructura aditiva de cambio y combinación y de la percepción hacia las matemáticas. La segunda, la realización de talleres que permitieran favorecer la construcción de número a partir de la manipulación de material concreto; la compresión del Sistema Numérico Decimal con el empleo de juegos y de material estructurado; y la resolución y construcción de problemas, dispuestos en la cotidianidad, que permitieran el análisis y la identificación de las vías de solución. Y como última etapa, los resultado obtenidos de la aplicación de la propuesta.

\section{Metodología}

El diseño empleado, es el cuasi experimental, por lo cual, se tomaron dos grupos uno de control y el otro experimental. Por lo tanto, en ambos grupos, se realizó el pretest de resolución de problemas y el de percepción hacia las matemáticas. Este trabajo, cuenta con la participación de 51 estudiantes del grado primero de primaria. El grupo está conformado por 26 niños y 25 niñas cuyas edades oscilan entre los 6 y 7 años.

\section{Hipótesis}

La aplicación del Sistema de Apoyo Pedagógico (SAP), favorece de manera pertinente en el desempeño de resolución de problemas en estructura aditiva, de manera particular los de cambio y combinación y los estudiantes tendrán una percepción agradable hacia las matemáticas.

\section{Caracterización de la muestra}

En este estudio participaron 50 estudiantes de los cursos del grado primero de primaria, de los cursos 104 y 106 jornada tarde, quienes se encuentran entre los 6 a 7 años de edad. Los dos grupos están conformados por un número similar de integrantes, en tanto el grupo 104 está constituido por 26 estudiantes y el grupo 106 por 25. La figura 1 muestra la distribución de integrantes de cada grupo según el género encontrando que en el grupo 104, el 54\% son niñas y el $46 \%$ niños, mientras que en el grupo 106 el $44 \%$ son niñas y el $56 \%$ niños.

Para definir los grupos se tuvieron en cuenta las variables como el logro académico, indicador principal e igualmente los resultados del pretest dado que aún cuando los resultados del pretest no arrojaron diferencias significativas entre los grupos el logro académico en la clase de matemáticas muestra resultados menos favorables para el grupo 106 seleccionado como grupo experimental. Por ende, el grupo 104 fue asumido como grupo control. De igual manera se observó el resultado del pretest agrado-des- 
agrado, muestran resultados similares entre los índices de percepción hacia las matemáticas. Por otra parte, se tuvieron en cuenta, otras situaciones que fueron pertinentes para la selección del grupo, entre estás, un cambio repetitivo de maestro en el curso 106 que no permitió una continuidad en el proceso de aprendizaje de este grupo, que no permitía un avance significativo en comparación con el otro grupo. Otro aspecto fue, la metodología empleada por la maestra del grupo 104, era similar a la propuesta presentada en este trabajo, por ende no tendría mucha relevancia ante los estudiantes.

\section{Resultados pretest de la prueba en resolución de problemas de estructura aditiva de cambio y combinación}

El pretest de la prueba en resolución de problemas, esta compuesta por cuatro problemas, dos de estructura aditiva de cambio y dos de combinación. Esta prueba tuvo el objetivo de identificar el estado inicial de los estudiantes de ambos grupos, tanto el de control como el experimental, para determinar cómo era su desempeño en la resolución de problemas de estructura aditiva.

Por lo tanto, mediante la aplicación del test se puedo reconocer si los estudiantes, comprendían el problema, si diseñaban y empleaban un plan adecuado para la resolución del problema y si la respuesta era la correcta. En la escala valorativa de esta prueba el puntaje máximo era el 18 y el puntaje mínimo era el 6 . Teniendo en cuenta las observaciones antes mencionadas.

La figura 2 muestra los resultados obtenidos de ambos grupos el de control y el experimental, en la prueba de pretest de resolución de problemas en particular los de tipo cambio. Por consiguiente, se evidencia que el grupo control el 42 $\%$ de los estudiantes obtuvieron un puntaje de 18 , que representa el más alto en la prueba por lo cual se demuestra un desempeño alto, mientras que el grupo experimental el $52 \%$ de los estudiantes tuvieron la misma puntuación. Sin embargo, en la gráfica, muestra que el grupo control, el $12 \%$ de los estudiantes obtuvieron el puntaje más bajo (6), y el grupo experimental el $16 \%$ de los estudiantes. Con respecto a lo anterior, se puede decir, que mientras que el grupo experimental, alcanzaron un nivel alto en la resolución de este tipo de problemas, a diferencia que el otro grupo.

La figura 3 se muestra los resultados obtenidos por ambos grupos, el de control y el experimental, en la prueba de resolución de problemas en particular en los problemas de estructura aditiva tipo combinación. Como se evidencia, el grupo control, el $58 \%$ de los estudiantes, lograron el puntaje más alto de la prueba (18) mientras que en el grupo experimental, fue equivalente al $52 \%$ de los estudiantes. Esto, quiere decir, que el grupo control alcanzó un nivel más alto en el desarrollo de estos problemas que el grupo experimental. Entre tanto, el grupo control, sólo el $4 \%$ de los estudiantes, obtuvieron el puntaje más bajo en la prueba (6) mientras que el grupo experimental en el $8 \%$ de los estudiantes.

\section{Pretest de la prueba de índice de agrado o des- agrado hacia las matemáticas}

Esta prueba, estuvo conformada por 6 preguntas, en las cuales, se reflejaba, el índice de los estudiantes, hacia las matemáticas; si le dan sentido para aprenderla y si les gusta o les parece muy difícil.

Para obtener los resultados, se estableció una escala valorativa, en la cual, 18 la determinaba la más alta puntuación, y 6 la más baja. Por lo cual en primera medida, se presentarán los resultados, que se obtuvieron del grupo de control, y seguidamente el grupo de experimentación.

La figura 4, muestra los resultados de los ambos grupos, el de control y el experimental, en la prueba de pretest de índice de agrado y desagrado hacia las matemáticas. Se evidencia, que el grupo de control, el 50\% de los estudiantes, manifiestan que tiene un nivel alto de agrado hacia las matemáticas, dado que arrojan el pun- 
Figura 1. Distribución según el género del grupo de control y de experimental
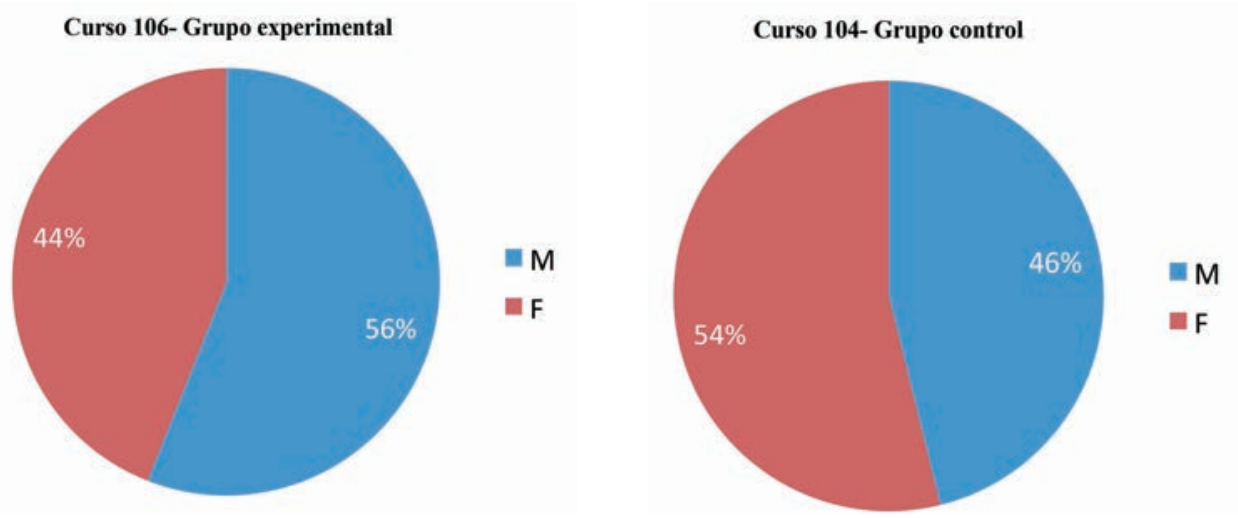

Fuente: interpretación de la autora.

Figura 2. Comparación de los ambos grupos, el de control y el de experimentación, en la prueba de pretest en resolución de problemas de estructura aditiva tipo cambio

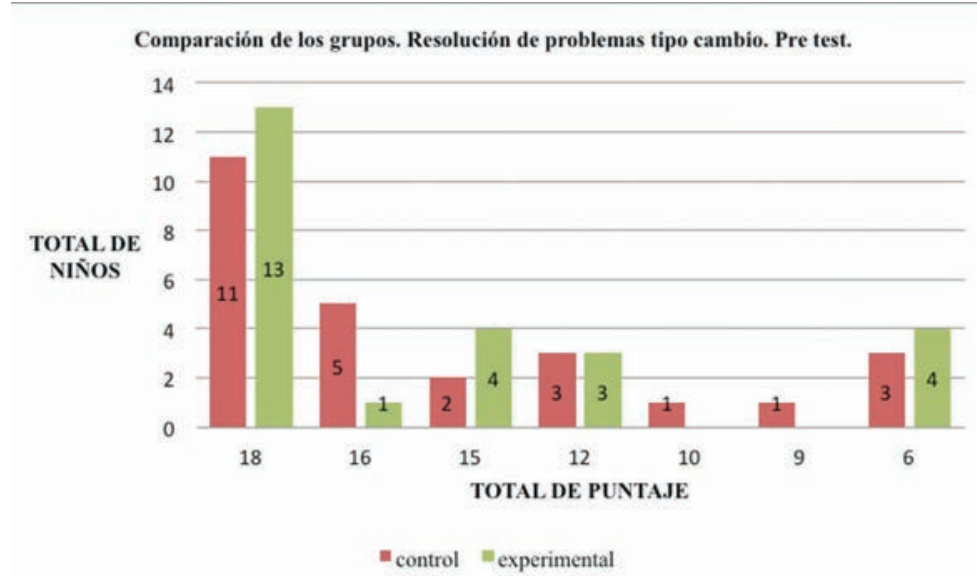

Fuente: interpretación de la autora.

Figura 3. Comparación de los ambos grupos, el de control y el de experimentación, en la prueba de pretest en resolución de problemas de estructura aditiva tipo combinación

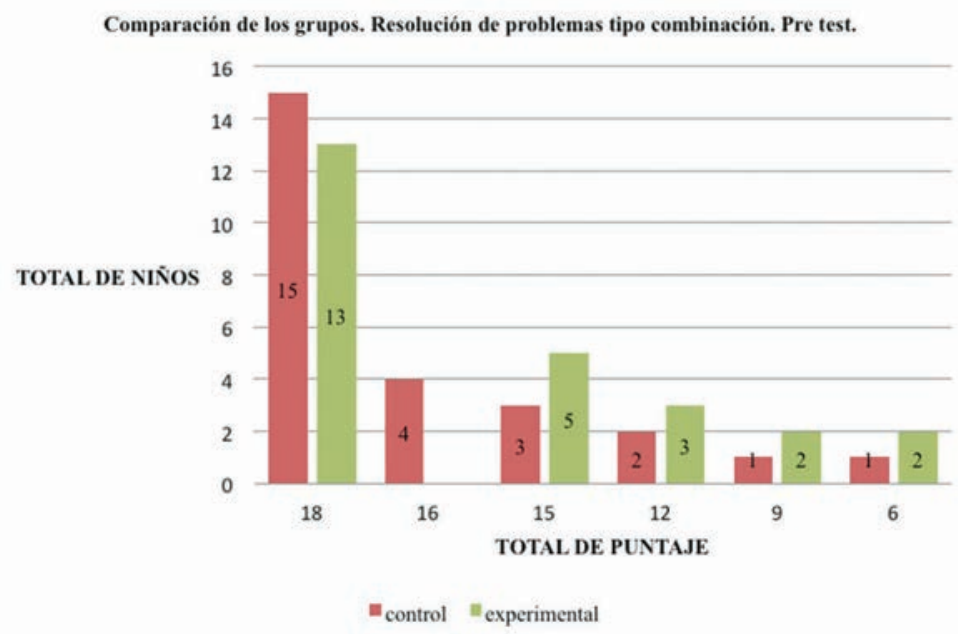

Fuente: interpretación de la autora. 
taje más alto de la prueba (18), mientras que en el grupo experimental, el $48 \%$ de los estudiantes, manifestaron lo mismo. Sin embargo en la figura, se muestra que ningún grupo arrojó un puntaje bajo, aunque es preciso decir que el grupo de control un $4 \%$ de los estudiante expresan un nivel medio de agrado hacia las matemáticas.

Postest de la prueba en resolución de problemas de estructura aditiva de cambio y combinación

La prueba postest consta de 4 problemas de estructura aditiva; 2 de tipo de cambio y 2 tipo combinación. Se aplica, después de haber implementado el proyecto de intervención pedagógica con el grupo experimental. La escala valorativa se determinó como el menor puntaje (6) y el mayor puntaje en la prueba es (18).

Esta prueba tuvo el objetivo de identificar el efecto que tuvo la implementación del Sistema de Apoyo pedagógico, sobre el desempeño de los estudiantes en la resolución de problemas de estructura aditiva en específico de cambio y combinación. Además permitiendo comparar los resultados obtenidos en ambos grupos, para poder analizar si efectivamente la propuesta pedagógica tuvo incidencia en el grupo experimental.

La figura 5, se encuentran los resultados de ambos grupo, el control y el experimental, de la prueba de postest de resolución de problemas de estructura aditiva en específico lo de cambio.

En el grupo control, se evidencia que sólo el $4 \%$ de los estudiantes obtuvieron el puntaje más alto de la prueba, mientras que en el grupo experimental el $16 \%$ de los estudiantes, lograron alcanzar un nivel alto. Por otra parte, en el grupo control, se evidencia, que el $15 \%$ de los estudiantes, obtuvieron el nivel más bajo de la prueba mientras que en el grupo experimental ningún participante arrojó ese resultado.
La figura 6 arroja los resultados obtenidos de ambos grupos control y experimental, en la prueba de postest en resolución de problemas de estructura aditiva en particular los de combinación.

Por lo cual se evidencia que, en el grupo control, el $15 \%$ de los estudiantes, alcanzaron un puntaje alto (18), en la resolución de este tipo de problemas, y el grupo experimental, el $36 \%$ de los estudiantes obtuvieron este mismo resultado, entre tanto el grupo experimental demuestra un porcentaje más alto que el presentado por el grupo control. Asimismo, en la figura se muestra que, en el grupo control, el $12 \%$ de los estudiantes obtuvieron el puntaje más bajo de la prueba (6), mientras que en el grupo experimental el $4 \%$ de los estudiantes.

Por otra parte, es notorio identificar que en la prueba del postest ambos grupos tuvieron un descenso en los resultados, siendo así que Karmiloff (1992) afirma que en su teoría del modelo de redescripción representacional, que abarca 3 fases recurrentes en el desarrollo. En la primera el niño centra su atención a la información que le proporciona el medio, permite que cree adiciones representacionales, y que finalice con cierta capacidad para actuar de manera correcta en relación a esas representaciones. Sin embargo en la fase 2 la autora comenta que "el estado actual de las representaciones que el niño tiene el conocimiento en un micro dominio predomina sobre la información procedente de los datos que viene de afuera" (p.39). En consecuencia, con los resultados arrojados, los estudiantes pueden estar en un descenso en que descuidan los detalles que les proporciona el ambiente y que hacen que a nivel conductual comentan ciertos errores, hasta tener un equilibrio tanto interno como externo.

\section{Postest de la prueba de índice de agrado o desagrado hacia las matemáticas}

La figura 7, muestra los resultados, obtenidos de la prueba de postest en la índice de 
Figura 4. Comparación de ambos grupos, el de control y el experimental, en la prueba de pretest en la percepción de agrado / desagrado

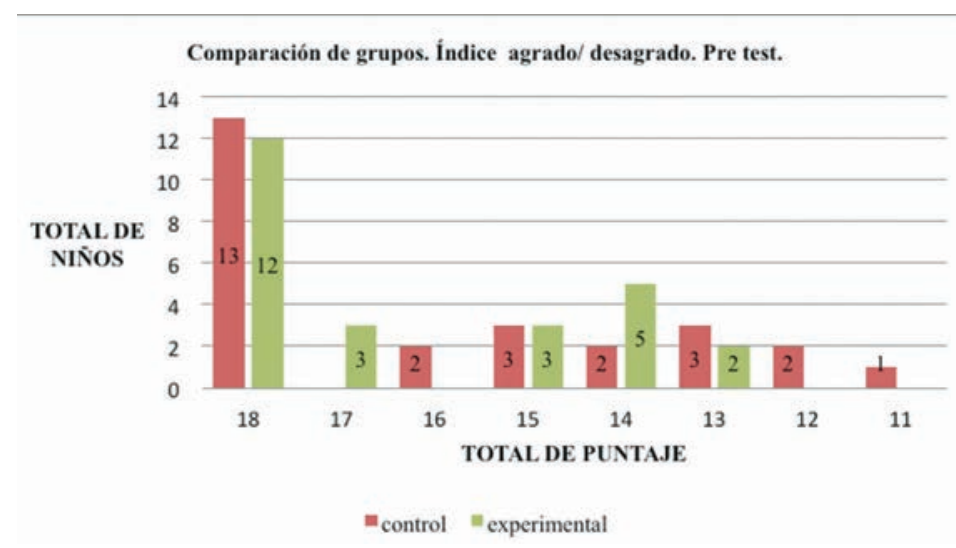

Fuente: interpretación de la autora.

Figura 5. Comparación de los ambos grupos, el de control y el de experimental, en la postest de resolución de problemas en estructura aditiva tipo cambio

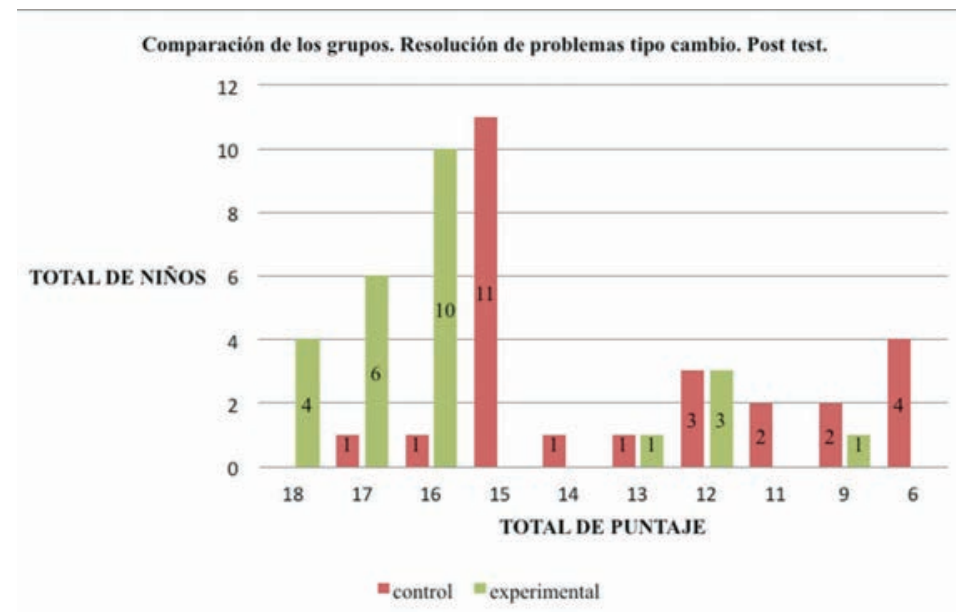

Fuente: interpretación de la autora.

Figura 6. Comparación de los ambos grupos, el de control y el de experimental, en la prueba de pos test de resolución de problemas en estructura aditiva tipo combinación

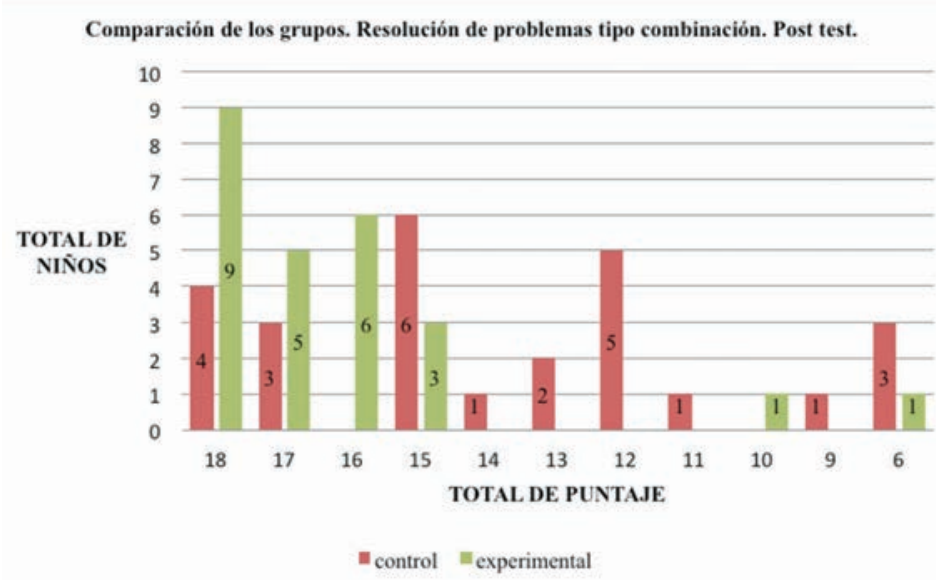

Fuente: interpretación de la autora. 
agrado y desagrado en el grupo control y en el experimental. Como se evidencia en la figura 7, el grupo control, el $35 \%$ de los estudiantes, manifestaron un agrado hacia las matemáticas, a diferencia del grupo experimental que muestra un $48 \%$ de estudiantes. Por otra parte, en el grupo control el $8 \%$ de los estudiantes, estimaron un nivel de desagrado hacia las matemáticas, mientras que en el grupo experimental no se evidencia puntajes bajos.

\section{Estadísticos descriptivos de los resultados pre y pos}

La tabla 2 muestra los puntajes promedios alcanzados por los dos grupos, el experimental y el control, en las pruebas pre y post en la prueba de resolución de problemas. Al observar los resultados, puede verse que el desempeño de los estudiantes en el pretest fue similar en los dos grupos en la resolución de problemas de cambio, teniendo 14,6 en el grupo control y 14,8 en el experimental. De ello puede decirse que el estado inicial de los grupos era similar antes de la aplicación del SAP en el grupo seleccionado.

En cuanto al desempeño de los estudiantes en los problemas de combinación, también en el pretest, puede verse que con una diferencia no muy significativa, 1,1 , el grupo control superó al experimental al tener un puntaje promedio de 16,1 en contraste con el 15 obtenido por el experimental (ver figura 8).

Posterior a la aplicación del SAP puede verse para los problemas de cambio en el postest el grupo experimental sobrepasa al experimental con un 15, 2 de promedio en los puntajes sobre un 12,1 en el puntaje obtenido por los integrantes del grupo control. De manera similar se evidencia en los resultados obtenidos en los problemas de combinación del pos test en donde se aprecia un promedio superior en el grupo experimental, 16, 1 sobre el puntaje promedio de 15 del grupo control.
La figura 8 muestra claramente el desempeño cercano, similar, de los dos grupos en la aplicación pre de la prueba de habilidades matemáticas: problemas de cambio y combinación, en contraste con el avance y aventajamiento que con respecto al grupo control muestra el grupo experimental en la aplicación pos test.

La tabla 3 muestra los puntajes promedio alcanzados en los grupos experimental y grupo control, en las pruebas pre y post de índice de agrado y desagrado hacia las matemáticas. Como se evidencia, en los resultados, el promedio del grupo control fue de 15,6 y el grupo experimental fue de 16,1 , teniendo una diferencia de 0,5.

Luego de la aplicación del SAP, se puede observar el grupo control obtuvo un 14,6 mientras que el grupo experimental alcanzó un puntaje de 15,9, teniendo una diferencia de 1,3 . Por lo tanto, se puede decir que aunque ambos grupos tiene similares resultados, en el índice de agrado y desagrado hacia las matemáticas, el grupo experimental sostuvo un puntaje alto, a diferencia del grupo control, que en el pre alcanzó un promedio de 15,6 a diferencia en el post que obtuvo un 14,6, teniendo una diferencia de 1.

La figura 9, se muestra la comparación de los grupos, de control y experimental, en los resultados de la prueba de índice de agrado y desagrado hacia las matemáticas, en la cual se observa que mientras el grupo experimental mantuvo el índice de agrado, mientras que el grupo control bajo su índice de agrado en la prueba post.

\section{Discusión}

Esta propuesta de intervención pedagógica, tuvo como propósito, favorecer el desempeño de la resolución de problemas, de estructura aditiva de tipo de combinación y cambio; desde una metodología basada en 
Figura 7. Comparación de los ambos grupos, el de control y el de experimental, en la prueba de postest en la índice de agrado / desagrado hacia las matemáticas

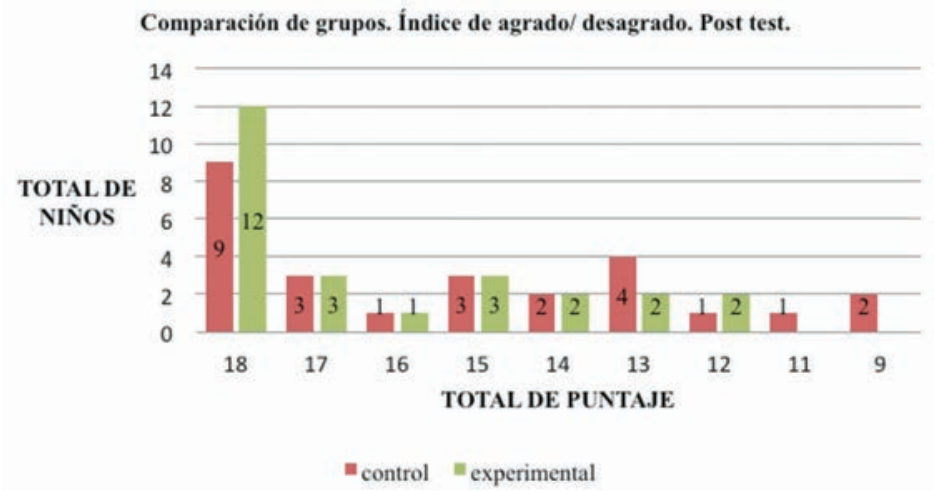

Fuente: interpretación de la autora.

Figura 8. Desempeño de los grupos en la prueba de habilidades matemáticas pre y postest

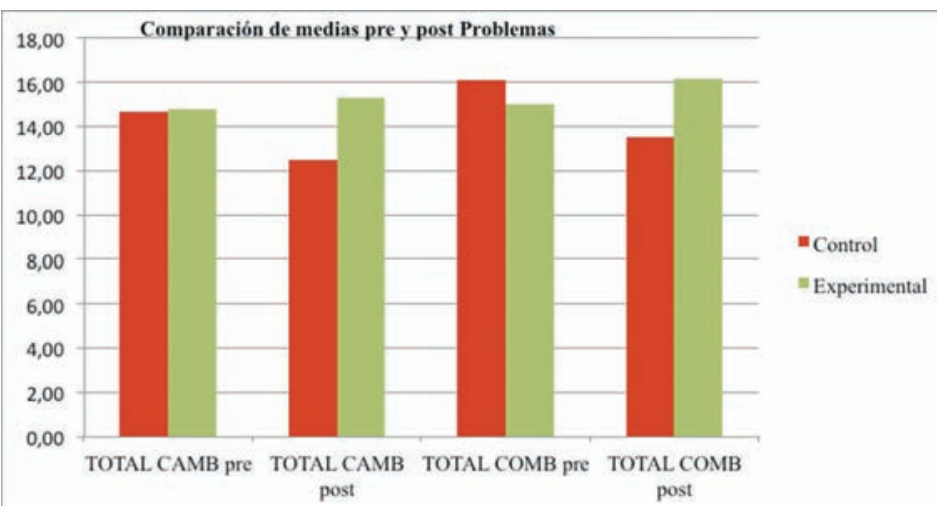

Figura 9. Índice de agrado / desagrado de los grupos en pre y postest

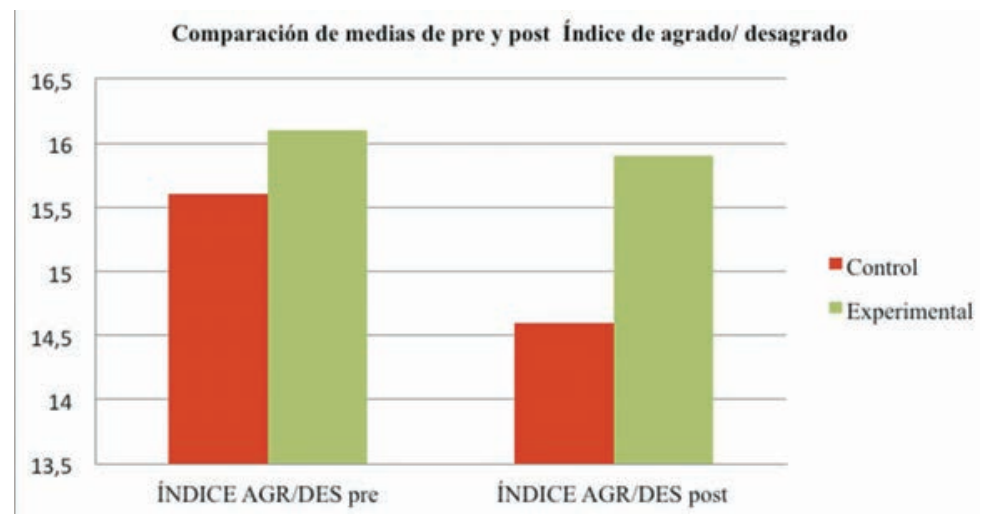

Tabla 2: Promedio de los grupo control y experimental en la prueba pre y post de resolución de problemas.

\begin{tabular}{ccccc}
\hline \multicolumn{4}{c}{ PUNTAJES PROMEDIO } \\
\hline Grupo & TOTAL CAMB pre & TOTAL CAMB post & TOTAL COMB pre & TOTAL COMB post \\
\hline Control & 14,6 & 12,5 & 16,1 & 13,5 \\
Experimental & 14,8 & 15,2 & 15 & 16,1 \\
\hline
\end{tabular}

Fuente: interpretación de la autora. 
los aportes de autores como Vigostky y Brunner, en relación a las estrategias de andamiaje y de apoyo, que permitan dar cuenta, que es importante fomentar el trabajo en equipo entre pares, para consolidar y asimilar conceptos, que no podían comprender con facilidad, pero con la ayuda de un tutor (maestro / par) pudieran entenderlo, para que posteriormente, lo realizaran solos. Por lo tanto, la clase de matemáticas, también tiene que ser un espacio, que permita el establecimiento de vínculos afectivos como el compañerismo, el trabajo cooperativo y la sana convivencia. De la misma manera, la propuesta se diseño con la finalidad que los estudiantes tuvieran una percepción de agrado, hacia las matemáticas, y por consiguiente manifestaran deseo por aprender y se mostraran más participativos y motivados

Durante la implementación de la propuesta de intervención se pudo evidenciar que, los estudiantes han incrementado el interés y la motivación por aprender. Además la confianza y el acercamiento entre la relación maestro y estudiantes, que permitió romper las barreras de indiferencia; dado así que los alumnos pudieran exponer sus preguntas y opiniones, de manera abierta sin sentirse presionados o excluidos.

Cabe recalcar, lo que afirmó Kammi (1995), en relación a la implementación de juegos porque son una herramienta muy útil en la enseñanza constructivista, ya que mediante estos los niños pueden desarrollar autonomía, independencia, autorregulación, y permite despertar el interés y la motivación. Es por lo tanto que el juego, no solo permite que las matemáticas se gocen y se consideren divertidas sino que también favorecen la compresión, la interacción social, el diálogo, la argumentación, la discusión, la proposición y el apoyo entre compañeros.

El apoyo y las estrategias de andamiaje, hacen que el estudiante, pueda acceder a un conocimiento más avanzado, por lo que fortalece la búsqueda de alternativas de soluciones ante cualquier dificultad. Por tanto, cuando los estu- diantes trabajan en grupo y se colaboran entre pares, hace que entre ellos se expliquen con mayor claridad temas, en términos más sencillos, siendo posible una asimilación más fácil de los conceptos. Esto también, pone en manifiesto, que los estudiantes, interactúen y se interrelacionen de manera más amena y compresible.

Por otra parte, Echenique (2006) define la resolución de problemas como la habilidad de la persona para planificar, adecuar y usar apropiadamente los conocimientos adquiridos. Por otro lado, Maza (1989) define la resolución de problemas, como"un principio didáctico fundamental en la enseñanza de la aritmética" (p.18). Por consiguiente para este autor es indispensable presentar situaciones problemáticas basadas en la vida cotidiana de los niños ya que esto permita que ellos establezcan relaciones entre las variables, y que los resuelvan a partir de sus propias estrategias. Así se orientan de manera precisa en los pasos propuestos por Polya (1989), como se describen a continuación:

1. Comprensión del problema: entender la situación, el tipo de información, decodificar el mensaje y pasarlo a un lenguaje matemático.

2. Concepción de un plan: se planifica las acciones, operaciones y el orden para cumplir una meta.

3. Ejecución del plan: en este paso se realiza el plan propuesto y se verifican los resultados que se obtuvieron y si la estrategia empleada fue la más adecuada o se necesita de otra.

4. Visión retrospectiva: revisión del proceso. Se contrasta el resultado con la meta esperada, además se reflexiona si hay otras vías de solución

De tal manera que el alumno pueda pasar de un nivel de hacer los problemas con ayuda, hasta un nivel en que los realice de manera independiente. 
Por otro lado, se resalta que, la utilización de material concreto y estructurado, permite que a los estudiantes se les facilite la construcción de su pensamiento lógico matemático, siendo así que, su aprendizaje sea más asequible y constructivo, y por lo tanto, se acomoda a su nivel de maduración y desarrollo cognitivo. Castro (2001) citando a Moisés Coriat, define los materiales didácticos y recursos en las matemáticas como objetos físicos utilizados para la enseñanza y el aprendizaje por tal razón deben identificar el uso que se hace de él y las funciones en las actividades con los estudiantes. De tal manera, que este autor identifica las dos maneras como se pueden utilizar los materiales didácticos y recursos: Los materiales didácticos se pueden utilizar de dos maneras:

- Partiendo del material planear las actividades que sean apropiadas para implementarlos

- Partiendo de la actividad ya planeada, recurrir a materiales adecuados.

Desde autores como Kammi (2000) y Cascallana (1999), las cuales afirman que el conocimiento lógico matemático, no puede ser trasmitido por explicación verbal; sino es por medio de las relaciones que construye internamente el niño, por medio de una abstracción reflexionante (construcción de relaciones entre objetos). Por lo tanto, la importancia de la manipulación de materiales no estructurados o estructurados. Entendiéndose a estructurados, como cualquier material variado, de fácil manipulación y que no sea tóxico que pueda ser empleado como medio didáctico para el aprendizaje de conceptos de matemáticos; y a los materiales no estructurados como todos los objetos no figurativos diseñados para la enseñanza de las matemáticas y la adquisición de conceptos. (Cascallana, 1999). Resnick y Ford (1998) citando a Zoltan Dienes, comprendió en sus estudios que la utilización de materiales hacen posible que se consoliden las estructuras matemáticas y el paso por un "ciclo de

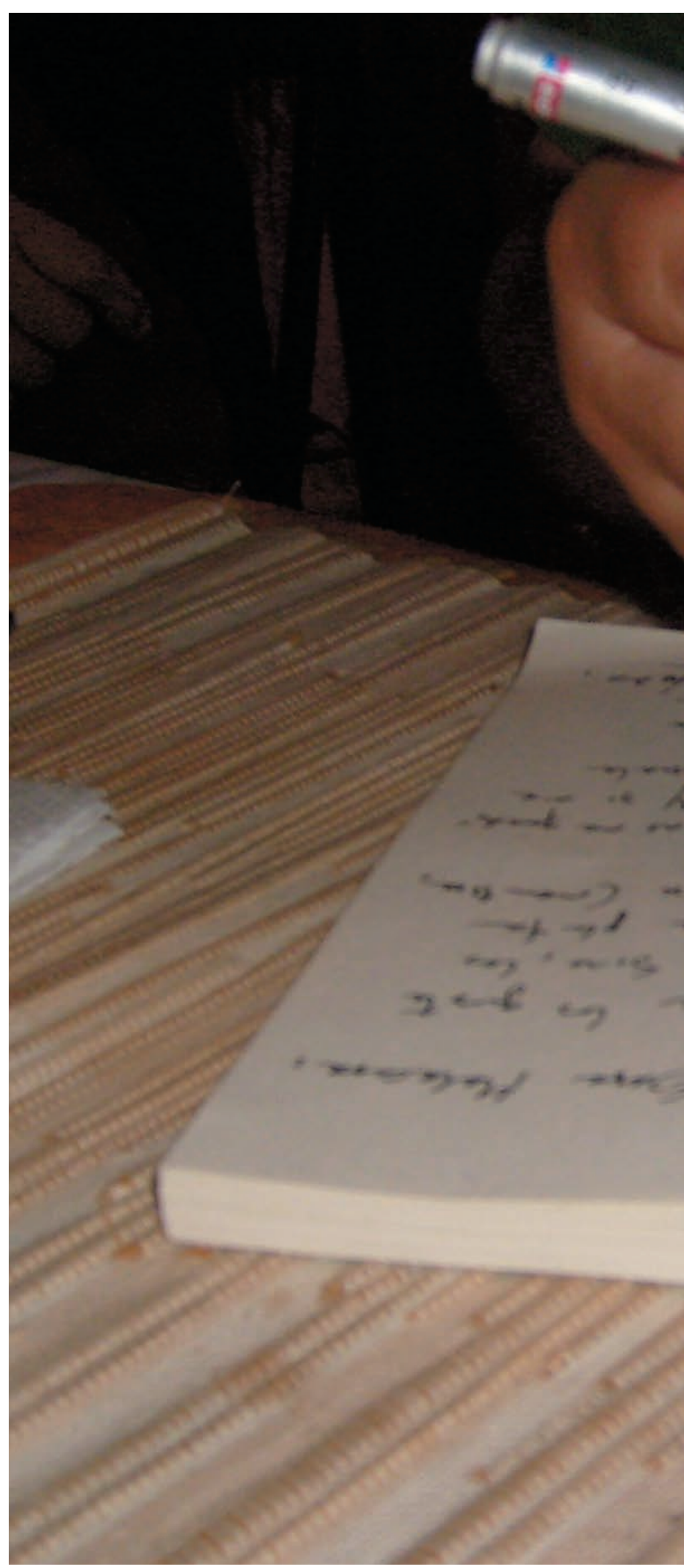




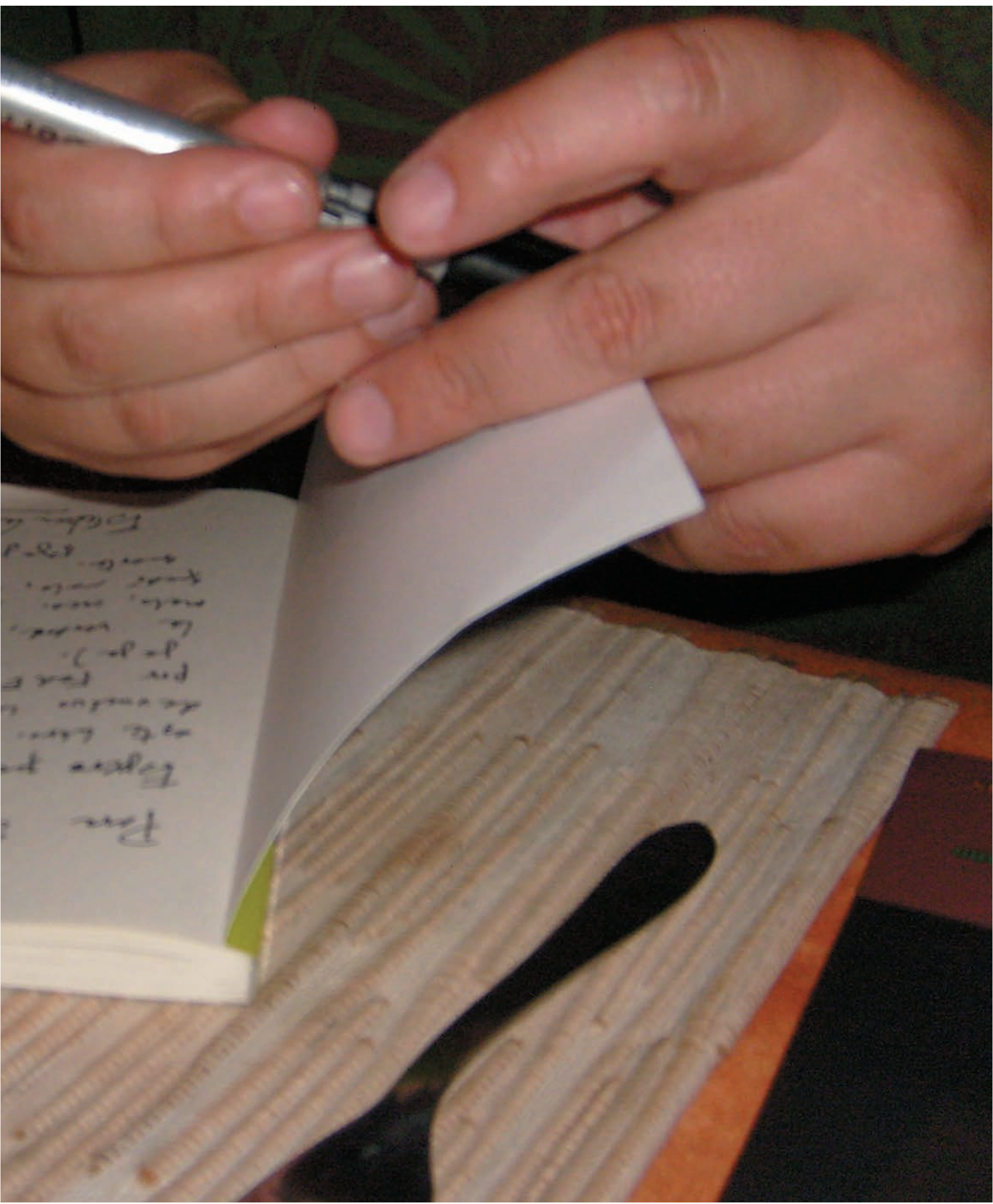


aprendizaje" apropiado desde lo concreto a lo simbólico.

Por consiguiente, cabe afirmar que sí es posible que mientras los estudiantes tengan experiencias con materiales, sean estructurados o no, pueden contribuir con una mayor compresión y sentido de las matemáticas, en relación a significancia de estas experiencias y a la interacción con su entorno.

Brunner citado por Resnick y Ford (1998, p.139) afirma que existen tres modos de representación: enactiva, icónica y simbólica. El primero se define como "un modo de representar eventos pasados mediante una respuesta motriz adecuada", es decir, mediante acciones con objetos concretos se hace posible una representación. El segundo modo de representación, es cuando se utilizan "imágenes mentales" es decir puede recordar sucesos y eventos en su mente. En el tercero, la simbólica, es a la utilización de símbolos que pueden atribuir significados, a sucesos, eventos, objetos, entre otros.

Asimismo, los estudiantes, fortalecen su lenguaje oral y escrito, en relación a la argumentación y la compresión de textos sencillos. Teniendo en cuenta, que cuando, a los estudiantes, se les muestra un problema de manera verbal, gráfica y escrita, los estudiantes tienen necesariamente hacer uso de sus representaciones mentales y de los procesos de lectura y escritura, para poder darle solución al problema.

En concordancia con la propuesta, se realizaron dos pruebas una de resolución de problemas y la otra de percepción hacia las matemáticas en dos momentos uno inicial, para tener conocimiento sobre el estado inicial de los estudiantes tanto en el grupo control como en el grupo de experimentación, y en un segundo momento un post para determinar el efecto de la propuesta con base a los resultados obtenidos en ambas pruebas.

En la prueba de pretest de resolución de problemas se analizaron los resultados obtenidos

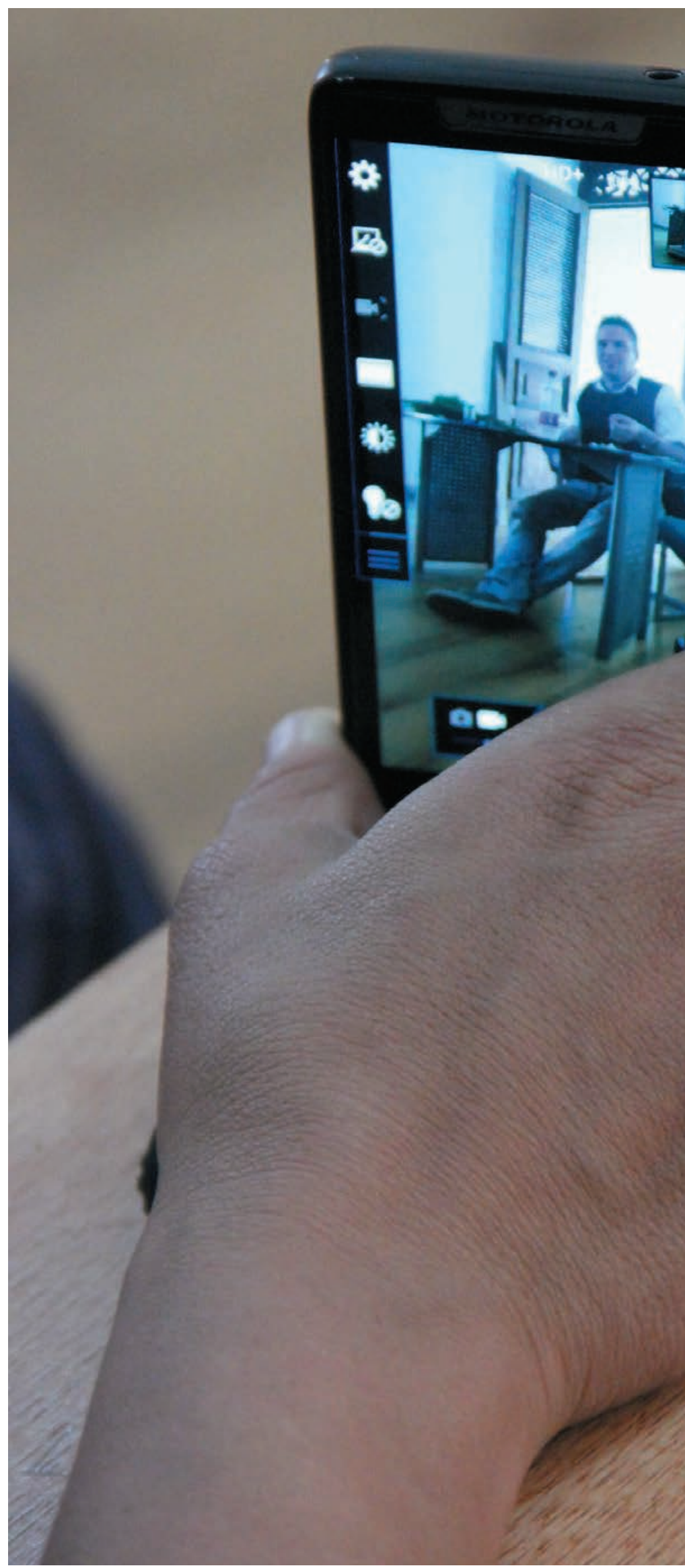


en cada tipo de problema. Entre tanto los resultados arrojados (ver figura 8), mostraron que en el grupo control, el puntaje promedio fue de 14,6 en los problemas de cambio, a diferencia del grupo experimental que obtuvo el 14, 8. De la igual manera en el grupo control en los problemas de tipo combinación, alcanzaron un promedio de 16, 1, mientras que el grupo experimental un 15. Como se puede observar entre ambos grupos en la prueba de pretest, tuvieron similares resultados y que no hay mayor diferencia de desempeño en ambos grupos.

En los resultados obtenidos del postest en la prueba de desempeño de resolución de problemas, se puede observar que en el grupo control alcanzó un promedio de 12,5 en contraste del grupo experimental con el 15, 2, por lo cual se evidencia una diferencia de 0,3. En los problemas de tipo combinación, el grupo control arrojó un puntaje promedio de 13,5 , en comparación al grupo experimental con el 16,1.

Con lo anterior, se puede decir, que el SAP, benefició al grupo experimental permitiendo que alcance un nivel alto en resolución de problemas en comparación al grupo control.

Con lo anteriormente dicho, en relación al pretest y postest en la prueba de resolución de problemas, es conveniente recalcar que, en el pretest ambos grupo mostraron resultados similares, y la diferencia entre ambos fue mínima. Sin embargo, en la prueba postest se evidencia que el grupo experimental sobrepasa al grupo control. Por lo cual se puede decir que aunque no es muy alta la diferencia entre ambos grupos en la prueba postest, la aplicación del SAP tuvo impacto en el desempeño en resolución de problemas en el grupo experimental.

Por otro lado, como se observa en la figura 9 los resultados arrojados en el pretest en el índice de agrado o desagrado en hacia las matemáticas, el grupo control tuvo un promedio de 15,6 a diferencia del grupo experimental con un 16, 1. En el postest, el grupo control obtuvo 
un promedio de 14,6 y el grupo experimental el 15,9 .

Con referente a los resultados, en la prueba de pre y post del índice de agrado o desagrado hacia las matemáticas, se puede concluir que los estudiantes del grupo control en la primera manifestaron un alto nivel de agrado, pero esto fue disminuyendo en la prueba post. Una de las posibles causas afirma Baroody (2005), es "las matemáticas escolares carecen de significado para los niños y no son nada divertidas, si es que no llegan a confundirles o amenazarles" ( $p$. 249). Entre tanto es indispensable que en la enseñanza de las matemáticas se tenga en cuenta los conocimientos informales y los intereses de los estudiantes, que permitan estimular el proceso de aprendizaje y además, que resulte relevante e importante para su comprensión. Por otro lado, el grupo experimental mantuvo el puntaje tanto en la prueba pre como en el post. Siendo que no se evidenció mayor relevancia en la aplicación del SAP, los estudiantes conservaron su índice de agrado, a pesar de que en la hipótesis se señalaba un aumento en la percepción de agrado.

\section{Conclusión}

Vigostky para el año 1978 expuso en su teoría socio cultural la necesidad de un apoyo de otra persona para su desarrollo, y en particular para alcanzar un nivel de zona próxima. Se puede afirmar, que es evidente, en el quehacer educativo, que se den espacios en los cuales los estudiantes, entre ellos se colaboren y se ayuden a comprender conceptos, y esto cambia la concepción de que el maestro tiene el poder total sobre el aula ; dado que como lo decía Baroody los estudiantes vienen con aprendizajes informales que permiten en la escuela formalizarlo, por lo cual cada estudiante viene con conocimientos que ayudan a constituir sus estructuras mentales, a partir de la interacción con el otro. Mediante la propuesta se puede evidenciar que la necesidad de grupo, y de escuchar al otro, favorece la convivencia, el respeto y la compresión del otro.

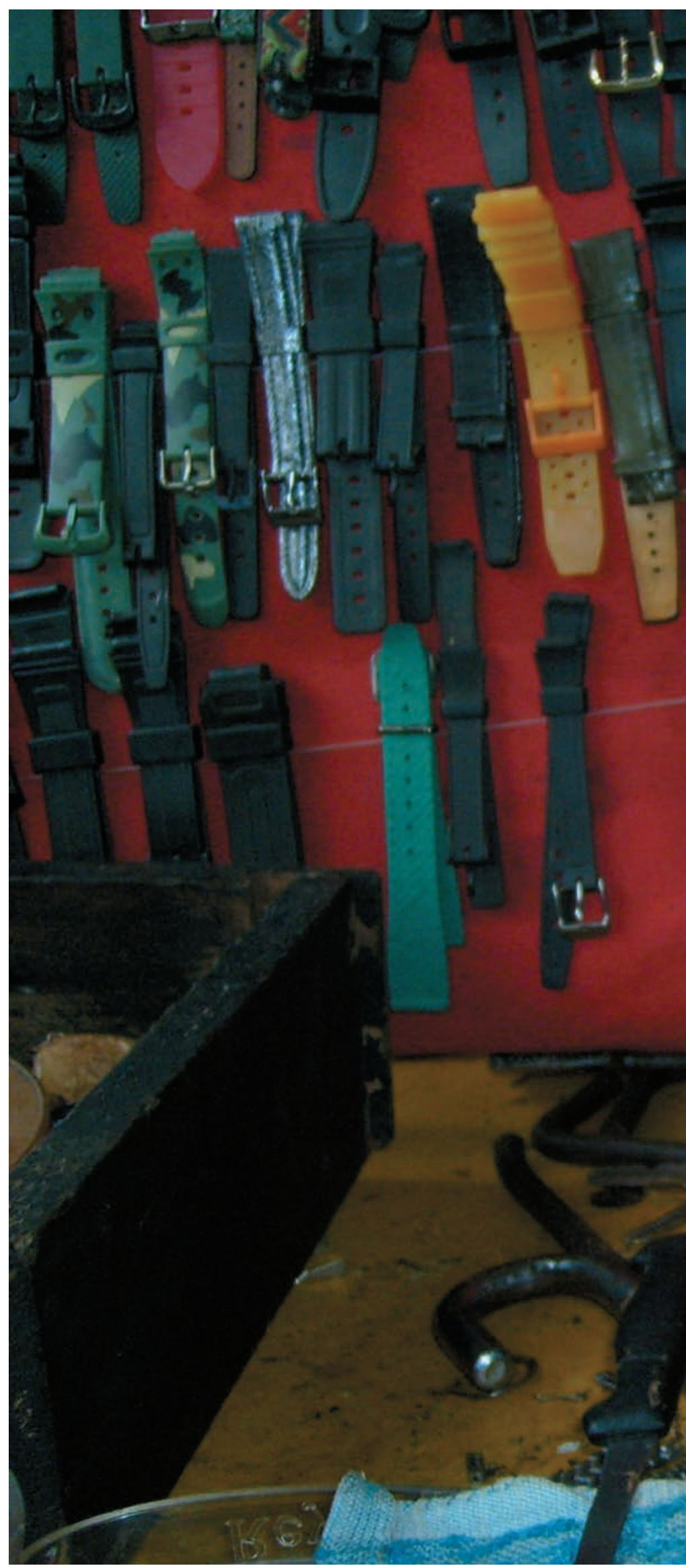


En la propuesta que se adelantó, se puede destacar que los estudiantes tuvieron un avance en la resolución de problemas de estructura aditiva en específico los de cambio y combinación, en relación al pretest. Se puede concluir que, en lo que refiera a las habilidades de resolución de problemas, se determina que cuando a los estudiantes se les proporciona diferentes situaciones problemáticas desde la oralidad, la gráfica y la escritura, a ellos se les facilita la interpretación de los problemas. Por otro lado es necesario que el maestro apoye a sus estudiantes, para poderlos orientar en el proceso propuesto por Polya (1989), para que se les facilite tanto la compresión, el diseño del plan, la ejecución del mismo y su posterior rectificación.

Además, se tiene en cuenta que a partir de la cotidianidad, desde la realidad particular del grupo de estudiantes, se puede contribuir al diseño de situaciones problemas, haciendo más significativo y relevante el sentido del aprendizaje de las matemáticas.

Cabe señalar, que los estudiantes, necesitan de unos insumos que permitan favorecer la resolución de problemas, en cuanto a la construcción de número y la consolidación del Sistema Numérico Decimal. El material facilitado hace posible que el alumno pueda utilizar y emplear los signos numéricos de manera oral y escrita de manera adecuada. Por lo cual, se puedo apreciar en los resultados del postest específicamente del grupo control, que se evidenció a pesar de que comprendían en su mayoría el problema, al ejecutar el procedimiento para dar respuesta no lo realizaban de manera correcta.

Finalmente, la utilización del lenguaje matemático en la comunicación de los sistemas de notación simbólica, la capacidad para la argumentación y la realización de conjeturas en el desarrollo de un problema fortaleció de manera transversal otras habilidades como la lectura y la escritura.

\section{Referencias bibliográficas}

Alcaldía mayor de Bogotá. (2011). Lo que dicen los resultados de las pruebas externas en Bogotá. Secretaría de educación del distrito. Recuperado de http://www. sedbogota.edu.co/evaluacion/images/archivos/serie_4/lo_que_dicen_resultados.pdf.

Baroody, J. (2005). El pensamiento matemático de los niños. Un marco Evolutivo para maestros de preescolar, ciclo inicial y educación especial. Madrid: Visor.

Cascallana, T. (1999). Iniciación a la matemática: materiales y recursos didácticos. Buenos Aires: Aula XXI Santillana.

Castro, E. (2001). Didáctica de las matemáticas en Educación Primaria. España: Síntesis.

Echenique, I. (2006), Matemáticas Resolución de Problemas, Educación Primaria. Gobierno de Navarra. Departamento de Educación. Recuperado de www.cfnavarra.es/publicaciones.

García, J. (2011) Educar desde la infancia: la tarea está pendiente. Diario el colombiano. Recuperado de http:// www.elcolombiano.com/BancoConocimiento/E/ educar_desde_la_infancia_la_tarea_esta_pendiente/educar_desde_la_infancia_la_tarea_esta_pendiente.asp

Karmiloff, A. (1992). Más allá de la modularidad. La ciencia cognitiva desde la perspectiva del desarrollo. Barcelona: Alianza editorial.

Kammi, C. (1995). Reinventado la aritmética III. España: Visor.

Kammi, C. (2000). El niño reinventa la aritmética. España: Visor.

Maza, C. (1995). Aritmética y Representación: de la comprensión del texto al uso de los materiales. Barcelona: Paidos.

Maza, C. (1989). Sumar y restar. Madrid: Visor.

Moll, L. (1993). Vigostky y la educación. Buenos Aires: Aique

Polya, G. (1989). Cómo plantear y resolver problemas. México: Editorial Trillas.

Renisck \& Ford. (1998). La enseñanza de las matemáticas y sus fundamentos psicológicos. España: Paidós.

Secretaria de Educación del Distrito. (2011). Lo que dicen los resultados de las pruebas externas. Bogotá: Autor 